\title{
Modeling stylized facts for financial time series
}

\author{
M.I. Krivoruchenko*,t), E. Alessio ${ }^{\dagger)}$, V. Frappietro ${ }^{\dagger}$, L.J. Streckert ${ }^{\dagger)}$ \\ *) Institute for Theoretical and Experimental Physics, B. Cheremushkinskaya 25 \\ 117259 Moscow, Russia \\ †) Metronome-Ricerca sui Mercati Finanziari, C. so Vittorio Emanuele 84 \\ 10121 Torino, Italy
}

\begin{abstract}
Multivariate probability density functions of returns are constructed in order to model the empirical behavior of returns in a financial time series. They describe the well-established deviations from the Gaussian random walk, such as an approximate scaling and heavy tails of the return distributions, long-ranged volatility-volatility correlations (volatility clustering) and return-volatility correlations (leverage effect). The model is tested successfully to fit joint distributions of the $100+$ years of daily price returns of the Dow Jones 30 Industrial Average.
\end{abstract}

Key words: time series, scaling, heavy tails, volatility clustering, leverage effect PACS: 89.65.Gh, 89.75.Da, 02.50.Ng, 02.50.Sk

The methods developed in studying complex physical systems have been successfully applied throughout decades to analyze financial data $[1,2,3]$ and they continue to attract gradual interest $[4,5,6,7,8,9,10]$. The field of research connected to modeling financial markets and development of statistically based real-time decision systems has recently been named Econophysics. In this paper, we construct a phenomenological model for description of the multivariate distribution of returns in a financial time series.

The random walk model proposed by Bachelier in the year 1900 [1] is equivalent to the Gaussian multivariate probability density function (PDF) of the returns $\xi_{i}$ :

$$
G_{n}\left(\xi_{i}\right)=\frac{1}{(2 \pi)^{n / 2}} \exp \left(-\frac{1}{2} \sum_{i=1}^{n} \sigma_{i}^{-2} \xi_{i}^{2}\right) \prod_{i=1}^{n} \frac{1}{\sigma_{i}} .
$$

The absence of the correlations, $\operatorname{Corr}\left[\xi_{i}, \xi_{j}\right]=0$, from a time frame greater 
than $t_{i}-t_{i-1}=20$ Min [6] has been widely documented and is often cited as a support for the efficient market hypothesis [8]. The multivariate PDFs constructed in this work are extensions of the Gaussian PDFs, aimed to model the well-established deviations in the behavior of financial time series from the Gaussian random walk.

The Lévy stable truncated univariate PDFs $[2,9]$ are known to provide, for a financial time series, (i) an approximate scaling invariance of the univariate PDFs with a slow convergence to the Gaussian behavior and (ii) the existence of heavy tails. We propose the multivariate Student PDFs,

$$
S_{n}^{\alpha}\left(\xi_{i}\right)=\frac{\Gamma\left(\frac{\alpha+n}{2}\right)}{(\alpha \pi)^{n / 2} \Gamma\left(\frac{\alpha}{2}\right)}\left(1+\frac{1}{\alpha} \sum_{i=1}^{n} \omega_{i}^{-2} \xi_{i}^{2}\right)^{-\frac{\alpha+n}{2}} \prod_{i=1}^{n} \frac{1}{\omega_{i}}
$$

for modeling the empirical PDFs with returns $\xi_{i}$. The marginal PDF (2) is again PDF (2). If we integrate out all of the $\xi_{i}$ except for one, we get (2) with $n=1$. The tails of the distributions behave empirically like [6] $\sim d \xi / \xi^{4}$, and so $\alpha \sim 3$.

For the Student PDF (2), we have $\mathrm{E}\left[\xi_{i}\right]=0$ and $\operatorname{Corr}\left[\left|\xi_{i}\right|,\left|\xi_{k}\right|\right]=2 /(2+\pi)=$ 0.39. The correlation of the returns vanishes as in the Gaussian random walk. The square of the volatility equals $\sigma_{i}^{2}=\operatorname{Var}\left[\xi_{i}\right]=\omega_{i}^{2} \alpha /(\alpha-2)$.

The cumulative returns, $\xi=\sum_{i=1}^{n} \xi_{i}$, are described by

$$
d W(\xi)=\int d \xi \delta\left(\xi-\sum_{i=1}^{n} \xi_{i}\right) S_{n}^{\alpha}\left(\xi_{i}\right) \prod_{i=1}^{n} d \xi_{i}=S_{1}^{\alpha}\left(\frac{\xi}{\Omega}\right) \frac{d \xi}{\Omega}
$$

where $\Omega^{2}=\sum_{i=1}^{n} \omega_{i}^{2}$. The variance of the $\xi$ increases linearly with $n$, in agreement with the Gaussian random walk and in the rough agreement with the empirical observations. Eq.(3) represents the scaling law for financial time series.

The multivariate Student PDFs have therefore heavy tails and the exact scaling invariance from the start. These distributions can be modified further to describe two other well-established stylized facts which are (iii) long ranged volatility-volatility correlations that are also known as volatility clustering [11] and (iv) return-volatility correlations that are also known as leverage effect $[12,13]$.

The empirical facts show that there is a slow decay of the correlation function. An extension of the $\operatorname{PDF}(2)$ that has the value $\operatorname{Corr}\left[\left|\xi_{i}\right|,\left|\xi_{j}\right|\right]$ which is decaying with time is rather straightforward. We use uncorrelated multivariate distributions for different groups of the returns. The analogy with the Ising model can be useful: The groups $\xi_{i}$ with the same multivariate Student PDF 
can be treated as domains of spins aligned in the same direction. We assign the usual probability for every such configuration

$$
w\left[\sigma_{1}, \ldots, \sigma_{n} \mid \beta\right]=N \exp \left(-\beta \sum_{i=1}^{n-1} \sigma_{i} \sigma_{i+1}\right)
$$

where $\sigma_{i}= \pm 1$. The normalization constant is given by $1 / N=2(2 \cosh (\beta))^{n-1}$. The correlation of the absolute values of the returns equals $2 /(2+\pi)$ provided that $\xi_{i}$ and $\xi_{k}$ belong to the same domain. Otherwise the result is zero. The probability of getting the $\xi_{i}$ and $\xi_{k}$ within the same domain can be found to be $w_{l}=e^{-\gamma l}$ where $e^{-\gamma}=e^{-\beta} /\left(e^{\beta}+e^{-\beta}\right)<1$ and $l=i-k$. The coefficient $\operatorname{Corr}\left[\left|\xi_{i}\right|,\left|\xi_{k}\right|\right]$ for the modified PDF has therefore the form $2 /(2+\pi) w_{l}$. The absence of the correlations would formally correspond to $\beta=+\infty(\gamma=+\infty)$. This is the case when the multivariate PDF is a product of the $n$ univariate PDFs.

In order to incorporate leverage effect, we consider the $\omega$-function depending on the signs $\epsilon_{i-p}=\operatorname{sign}\left(\xi_{i-p}\right)$ of the lagged returns $(p=1,2, \ldots)$. The values $\epsilon_{i}= \pm 1$ are assumed to be independent variables which take the two values \pm 1 with the equal probabilities. The $\epsilon_{i}$ dependence is modeled by $\omega_{i}=C\left(1-\rho \frac{1-\nu}{\nu} \sum_{p=1}^{\infty} \nu^{p} \epsilon_{i-p}\right)^{2}$ where $C$ is a normalization factor. Negative recent returns $\epsilon_{i-p}=-1(p=1,2, \ldots)$ increase the volatility, so $\rho>0$. The value of $\rho$ is connected to the overall strength of leverage effect.

Taking volatility clustering and leverage effect into account, we obtain for $l=i-k>0$ :

$$
\begin{aligned}
\operatorname{Corr}\left[\left|\xi_{i}\right|,\left|\xi_{k}\right|\right] & =\frac{2}{\pi} \frac{\left(1-\frac{2}{\pi}\right) g_{l} w_{l}+\frac{2}{\pi}\left(g_{l}-1\right)}{g_{0}-\frac{4}{\pi^{2}}} \\
\operatorname{Corr}\left[\left|\xi_{i}\right|, \xi_{k}\right] & =\frac{2}{\pi} \frac{\left(1-\frac{2}{\pi}\right) w_{l}+\frac{2}{\pi}}{\sqrt{\left(g_{0}-\frac{4}{\pi^{2}}\right) g_{0}}} h_{l}
\end{aligned}
$$

where $g_{l}=\mathrm{E}\left[\omega_{i} \omega_{k}\right]$ and $h_{l}=\mathrm{E}\left[\omega_{i} \epsilon_{k} \omega_{k}\right]$ can be calculated analytically.

The empirical correlation functions for the $100+$ years of daily price returns of the Dow Jones 30 Industrial Average are fitted using a superposition $w_{l}=$ $c_{1} e^{-\gamma_{1} l}+c_{2} e^{-\gamma_{2} l}$ for $l>0$ with $c_{1}=0.18, c_{2}=0.08, \gamma_{1}=1 / 1200$, and $\gamma_{2}=1 / 233$ (else $c_{0}=0.74$ and $\gamma_{0}=+\infty$, so that $\sum_{m=0}^{2} c_{m}=1$ ). We have also used $\rho=1$ and $\nu=\exp (-1 / 16)$. The results are in a good agreement with the data. The quantitative comparison can be found in Ref. [14]. The value of $c_{1}+c_{2}=0.26$ indicates that $26 \%$ of the empirical PDF consists of products of the multivariate Student PDFs. The value $\operatorname{Corr}\left[\xi_{i},\left|\xi_{k}\right|\right]$ vanishes at $i>k$ in agreement with the observations, since the $\omega_{i}$ depend on lagged returns only. 
The complete multivariate PDF of the returns is given by

$$
\begin{aligned}
S_{n}^{\alpha}\left(\xi_{n}, \ldots, \xi_{1}\right)_{C}= & \sum_{m=0}^{2} c_{m} \sum_{s=0}^{n-1} \frac{\exp \left(-\beta_{m}(n-2 s-1)\right)}{\left(2 \cosh \left(\beta_{m}\right)\right)^{n-1}} \\
& \times \sum_{n_{s}, \ldots, n_{0}} \prod_{f=1}^{s+1} S_{n_{f}-n_{f-1}}^{\alpha}\left(\xi_{n_{f}-1}, \ldots, \xi_{n_{f-1}}\right)
\end{aligned}
$$

where $n_{s+1}=n+1, n \geq n_{s}>\ldots>n_{1} \geq 2$, and $n_{0}=1$. The marginal probability of the $\operatorname{PDF}(7)$ is a $\operatorname{PDF}(7)$ again.

The authors wish to thank the Dow Jones Global Indexes for providing historical prices of the Dow Jones Averages. M.I.K. is grateful to Metronome-Ricerca sui Mercati Finanziari for kind hospitality.

\section{References}

[1] L. Bachelier, Ann. Sci. Ercole Norm. Suppl. 3, 21 (1900).

[2] P. Levy, Théorie de l'Addition des Variables Aléatoires (Gauthier-Villars, Paris, 1937).

[3] B. B. Mandelbrot, J. Business 36, 294 (1963).

[4] R. Cont and D. Sornette, J. Phys. I France 7, 431 (1997).

[5] N. Vandewalle and M. Ausloos, Physica A 246454 (1997).

[6] P. Gopikrishnan, V. Plerou, L. A. Nunes Amaral, M. Meyer, and H. E. Stanley, Phys. Rev. E60, 5305 (1999).

[7] E. Alessio, A. Carbone, G. Castelli, and V. Frappietro, The European Physical J. B27, 197 (2002).

[8] E. F. Fama, Journal of Finance 25, 383 (1970).

[9] R. N. Mantegna and H. E. Stanley, Nature 376, 46 (1995).

[10] K. Urbanowicz and J. A. Holyst, Phys. Rev. E67, 046218 (2003).

[11] Z. Ding, C. W. J. Granger and R. F. Engle, J. Empirical Finance 1, 83 (1993).

[12] F. Black, Proceedings of the $19^{7} 6$ Americal Statistical Association, Business and Economical Statistics Section, p. 177 (1976).

[13] J. C. Cox and S. A. Ross, J. Fin. Eco. 3, 145 (1976).

[14] E. Alessio, V. Frappietro, M. I. Krivoruchenko, and L. J. Streckert, http://arXiv.org/abs/cond-mat/0310300. 


\title{
Return Distributions in Financial Time Series
}

with application to pricing derivatives and risk management

E. Alessio, V. Frappietro, M. I. Krivoruchenko, L. J. Streckert

Metronome-Ricerca sui Mercati Finanziari, Torino

\author{
November 15, 2003 \\ APFA4, Warsaw, Poland
}

- Empirical deviations from the Gaussian random walk:

(i) approximate scaling,

(ii) heavy tails of the return univariate distributions,

(iii) long ranged volatility-volatility correlations (volatility clustering),

(iv) return-volatility correlations (leverage effect).

४ Multivariate Student PDFs for modeling (i) - (iv).

४ Conditional and marginal PDFs.

$\downarrow$ Pricing Log contracts. 


\section{Why Econophysics?}

Methods developed in studying complex physical systems have been successfully applied through decades to analysis of financial data

L. Bachelier, Ann. Sci. Ercole Norm. Suppl. 3, 21 (1900),

P. Levy, Théorie de l'Addition des Variables Aléatoires

(Gauthier-Villars, Paris, 1937),

B. B. Mandelbrot, J. Business 36, 294 (1963),

R. N. Mantegna and H. E. Stanley, Nature 376, 46 (1995) and continue to attract gradual interest.

Last years:

Development of statistically based real-time decision systems results in the growing importance of the Econophysics research.

Over $10 \%$ of the papers in Physica $\mathbf{A}$ are related to Econophysics.

New journals: Quantitative Finance, .... 
Financial time series are described by a generalized Wiener process:

$$
\frac{\mathbf{d} \mathbf{S}(\mathbf{t})}{\mathbf{S}(\mathbf{t})}=\mu \mathbf{d t}+\mathbf{d} \xi(\mathbf{t}) .
$$

Here, $d \xi(t)$ is a noise with the expectation value and variance

$$
\mathrm{E}[\mathbf{d} \xi(\mathbf{t})]=\mathbf{0}, \quad \operatorname{Var}[\mathbf{d} \xi(\mathbf{t})]=\sigma(\mathbf{t})^{\mathbf{2}} \mathbf{d t} \mathbf{t} .
$$

The volatility $\sigma(t)$ is a measure of the magnitude of market fluctuations.

We consider a discrete random walk and set

$$
\mathbf{d t} \equiv \mathbf{t}_{\mathbf{i}}-\mathbf{t}_{\mathbf{i}-\mathbf{1}}, \quad \mathbf{S}_{\mathbf{i}} \equiv \mathbf{S}\left(\mathbf{t}_{\mathbf{i}}\right), \quad \xi_{\mathbf{i}} \equiv \mathbf{d} \xi\left(\mathbf{t}_{\mathbf{i}}\right), \quad \sigma_{\mathbf{i}} \equiv \sigma\left(\mathbf{t}_{\mathbf{i}}\right) .
$$


The random walk model proposed by Bachelier in $\mathbf{1 9 0 0}$ year is equivalent to the Gaussian multivariate PDF:

$$
\mathbf{G}_{\mathbf{n}}\left(\xi_{\mathbf{i}}\right)=\frac{1}{(2 \pi)^{\mathbf{n} / 2}} \exp \left(-\frac{1}{2} \sum_{\mathrm{i}=1}^{\mathrm{n}} \sigma_{\mathrm{i}}^{-2} \xi_{\mathbf{i}}^{2}\right) \prod_{\mathrm{i}=1}^{\mathrm{n}} \frac{1}{\sigma_{\mathbf{i}}}
$$

No statistically significant correlations at $t_{i}-t_{i-1}>20 \mathrm{Min}$

$$
\operatorname{Corr}\left[\xi_{\mathbf{i}}, \xi_{\mathbf{j}}\right]=\delta_{\mathbf{i j}}
$$

The multivariate PDFs constructed in this work are extensions of the Gaussian PDFs to model the well established deviations in the behavior of financial time series from the Gaussian random walk:

(i) heavy tails of the return distributions,

(ii) approximate scaling,

(iii) volatility clustering, and

(iv) leverage effect. 
Deviations from Gaussian random walk: Autocorrelations

Autocorrelation function of $\xi(t)$ and autocorrelation function of $|\xi(t)|$, with sampling time interval $\Delta t=1 \mathrm{Min}$.
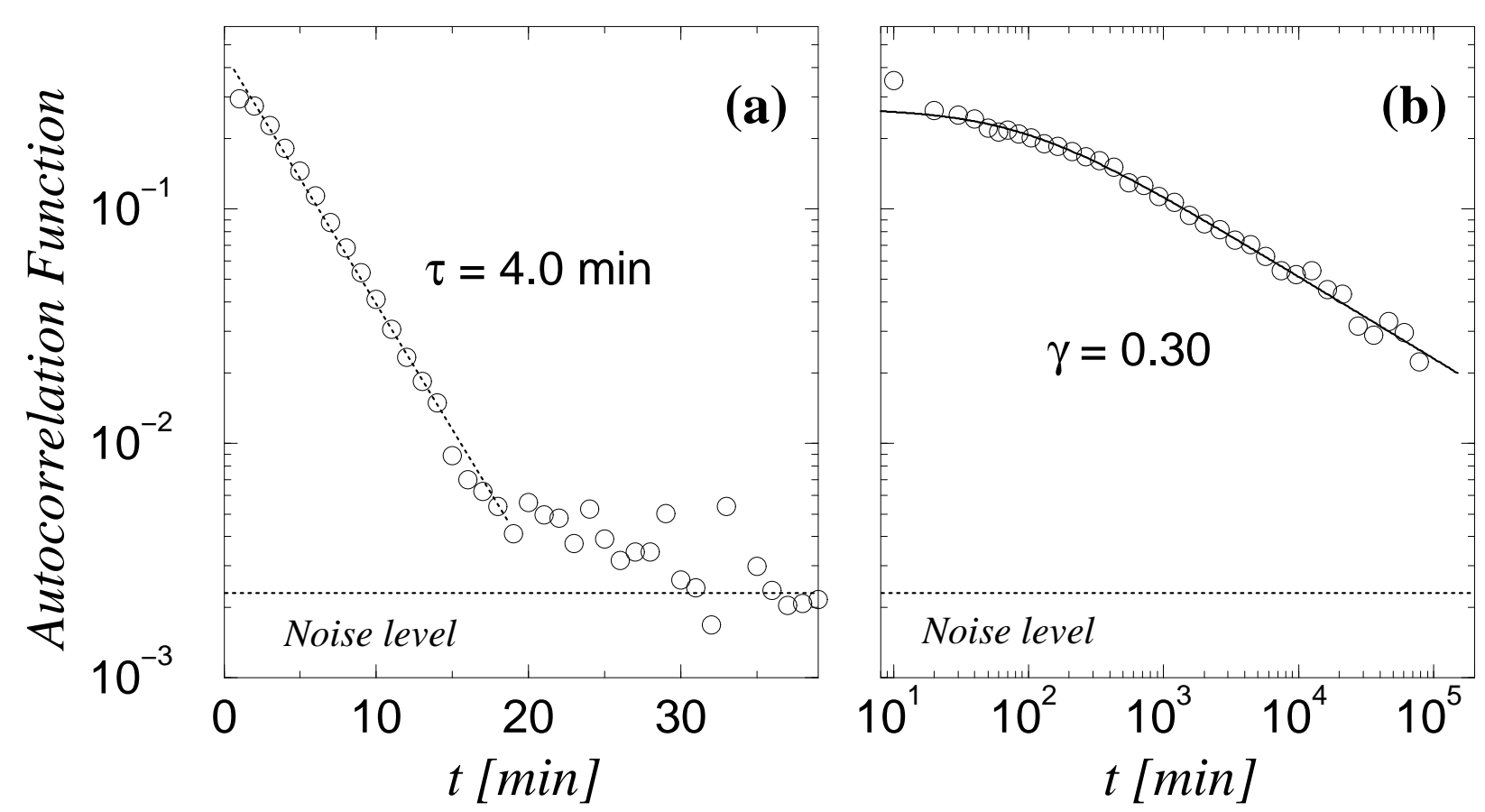

Results are from: Yanhui Liu, Parameswaran Gopikrishnan, Pierre Cizeau, Martin Meyer, Chung-Kang Peng, H. Eugene Stanley,

E-print arXiv: cond-mat/9903369 (1999). 


\section{Deviations from Gaussian random walk: Heavy tails}

Probability density of $\Delta t=5$ minutes increments of S\&P 500 index future prices compared to the Gaussian distribution for the same mean and variance. The data set contains 75000 prices.

Probability density of price changes

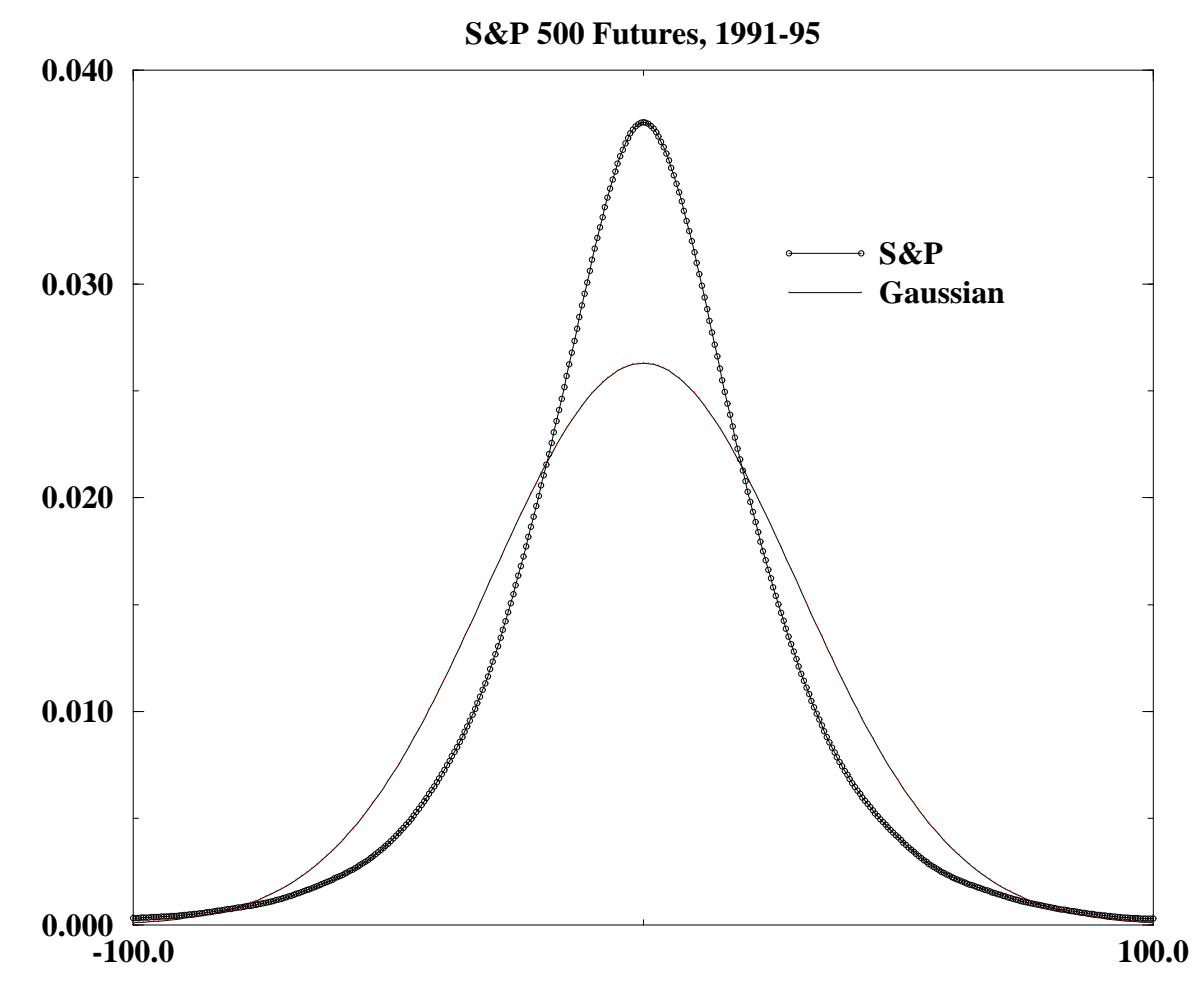

Results are from: R. Cont, E-print arXiv: cond-mat/9705075 (1997). 
Deviations from Gaussian random walk: Heavy tails

Comparison with the empirical data:

$100+$ years of daily quotes of the DOW Jones 30 Industrial

Average

The $\alpha=3$ Student and Gaussian univariate PDFs as compared to the hystogram for the DJIA.

The value of $\xi$ is the increment (noice) added to the path followed by the index value, $\sigma^{2}=\operatorname{Var}[\xi]$. The common scale is fixed by fitting the variance. The solid line describes the Student PDF, the short-dashed line corresponds to the Gaussian PDF. 


\section{Deviations from Gaussian random walk: Leverage effect}

The leverage effect in the DOW Jones 30 Industrial Average from 1900 until 2000 year

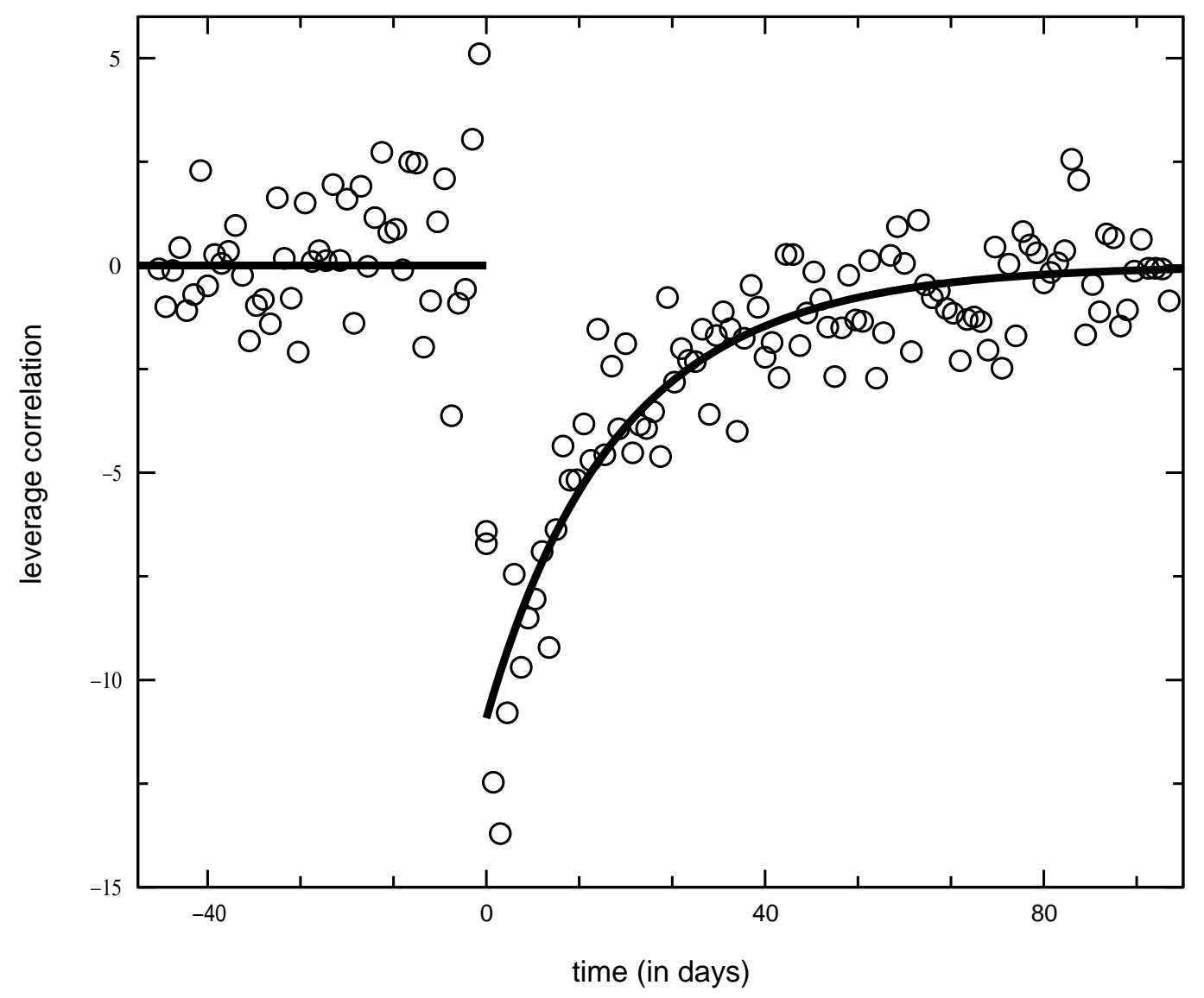

Results are from: Josep Perello, Jaume Masoliver,

E-print arXiv: cond-mat/0111334 (2001). 
Lévy stable truncated PDFs proposed by Mantegna and Stanley (1995) are known to provide (i) approximate scaling and (ii) heavy tails. Two other stylized facts (iif) volatility clustering and (iv) leverage effect

. Therefore

\section{MULTIVARIATE STUDENT PDF}

to model realistic distribution of returns:

$$
\mathbf{S}_{\mathbf{n}}^{\alpha}\left(\xi_{\mathbf{i}}\right)=\frac{\boldsymbol{\Gamma}\left(\frac{\alpha+\mathbf{n}}{2}\right)}{(\alpha \pi)^{\mathbf{n} / \mathbf{2}} \Gamma\left(\frac{\alpha}{2}\right)}\left(1+\frac{1}{\alpha} \sum_{\mathbf{i}=1}^{\mathbf{n}} \omega_{\mathbf{i}}^{-\mathbf{2}} \xi_{\mathbf{i}}^{\mathbf{2}}\right)^{-\frac{\alpha+\mathbf{n}}{2}} \prod_{\mathbf{i}=1}^{\mathbf{n}} \frac{1}{\omega_{\mathbf{i}}}
$$

The marginal Student PDF is again a Student PDF. If we integrate out all the increments $\xi_{i}$ except for a one, we get (\#) with $n=1$. The tails of the distributions behave empirically like $\sim d \xi / \xi^{4}$, and so $\alpha \sim 3$ 
We compare:

$\checkmark$ The $n=1, \alpha=3$ Student PDF,

$\checkmark$ The Gaussian PDF, and

$\checkmark$ The empirical univariate PDF.

NB: The empirical PDF is constructed for the $100+$ years of the daily quotes of the DOW Jones 30 Industrial Average for a period of 26.05.1896 - 31.12.1999 (totally 28507 working days)

The drift rate and variance are found to be

$$
\mu=\mathbf{0 . 0 0 0 2 5 7} \& \quad \operatorname{Var}[\xi]=\mathbf{0 . 0 0 0 1 1 7}
$$

The PDFs are shown as functions of the $\xi / \sigma$ with $\sigma^{2}=\operatorname{Var}[\xi]$. 
Deviations from Gaussian random walk: Heavy tails

Comparison with the empirical data:

$100+$ years of daily quotes of the DOW Jones 30 Industrial

Average

The $\alpha=3$ Student and Gaussian univariate PDFs as compared to the hystogram for the DJIA.

The value of $\xi$ is the increment (noice) added to the path followed by the index value, $\sigma^{2}=\operatorname{Var}[\xi]$. The common scale is fixed by fitting the variance. The solid line describes the Student PDF, the short-dashed line corresponds to the Gaussian PDF. 
Student random walk: Autocorrelations

For Student PDF, we have

$$
\mathrm{E}\left[\xi_{i}\right]=0 \quad \& \quad \operatorname{Var}\left[\xi_{i}\right]=\omega_{i}^{2} \frac{\alpha}{\alpha-2},
$$

while the autocorrelation functions have the form

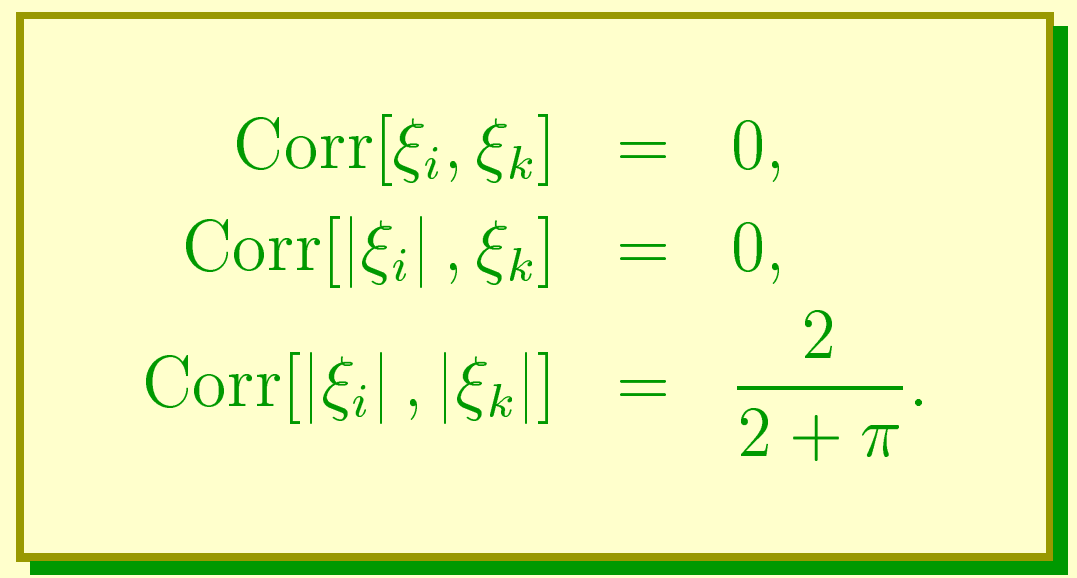

The square of the volatility equals

$$
\sigma_{\mathrm{i}}^{2}=\omega_{\mathrm{i}}^{2} \frac{\alpha}{\alpha-2}
$$

The correlation function of $\left|\xi_{i}\right|$ is positive and does not depend on the $\operatorname{lag} l=i-j$. 
Student random walk: Exact scaling

If all components of the vectors $\psi=\left(\psi_{1}, \ldots, \psi_{n}\right)$ and $\eta=\left(\eta_{1}, \ldots, \eta_{\alpha}\right)$ $\sim N(0,1)$, a random vector

$$
\xi_{\mathbf{i}}=\omega_{\mathbf{i}} \psi_{\mathbf{i}}\left|\frac{\alpha}{\eta^{2}}\right|^{\frac{1}{2}}
$$

with $\eta=|\eta|$ has the Student PDF.

The cumulative effect, $\xi=\sum_{i=1}^{n} \xi_{i}$, is described by

$$
\mathrm{d} \mathbf{W}(\xi)=\int \mathrm{d} \xi \delta\left(\xi-\sum_{\mathbf{i}=1}^{\mathbf{n}} \xi_{\mathbf{i}}\right) \mathbf{S}_{\mathbf{n}}^{\alpha}\left(\xi_{\mathbf{i}}\right) \prod_{\mathbf{i}=1}^{\mathbf{n}} \mathrm{d} \xi_{\mathbf{i}}=\mathbf{S}_{1}^{\alpha}\left(\frac{\xi}{\Omega}\right) \frac{\mathrm{d} \xi}{\Omega}
$$

where $\Omega^{2}=\sum_{i=1}^{n} \omega_{i}^{2}$. The value $\xi$ can be represented as

$$
\xi=\left(\omega_{1} \psi_{1}+\omega_{\mathbf{2}} \psi_{\mathbf{2}}+\ldots+\omega_{\mathbf{n}} \psi_{\mathbf{n}}\right)\left|\frac{\alpha}{\eta^{2}}\right|^{\frac{1}{2}} \sim \Omega \psi\left|\frac{\alpha}{\eta^{2}}\right|^{\frac{1}{2}}
$$

where $\psi \sim N(0,1)$. The variance of the $\xi$ increases linearly with $n$, in agreement with the empirical observations. We got the scaling law, observed empirically by Mandelbrot (1963). 
The Student conditional PDF at $n \gg l=n-k$ that gives a forecast density $l$ steps ahead:

$$
\mathbf{S}_{\mathbf{n}}^{\alpha}\left(\xi_{\mathbf{n}}, \ldots, \xi_{\mathbf{k}+1} \mid \xi_{\mathrm{k}}, \ldots, \xi_{1}\right)=\frac{\mathbf{S}_{\mathbf{n}}^{\alpha}\left(\xi_{\mathrm{n}}, \ldots, \xi_{1}\right)}{\mathbf{S}_{\mathrm{k}}^{\alpha}\left(\xi_{\mathrm{k}}, \ldots, \xi_{1}\right)} \sim \frac{1}{\left(2 \pi v^{2}\right)^{1 / 2}} \exp \left(-\frac{1}{2 v^{2}} \sum_{\mathrm{i}=\mathrm{k}+1}^{\mathrm{n}} \xi_{\mathrm{i}}^{2}\right)
$$

where

$$
v^{2}=\frac{1}{k} \sum_{i=1}^{k} \xi_{i}^{2}
$$

The conditional PDF of the values $\xi_{n}, \ldots, \xi_{k+1}$ is close to the Gaussian PDF.

The ARCH models: The increments are distributed as $\sim N\left(0, v^{2}\right)$, with $v$ being a function of the lagged increments. The estimator (1) is one of the possible estimators quantifying the volatility within the ARCH framework. 


The multivariate Student PDFs have heavy tails, the ex-
act scaling invariance, and volatility clustering from the
start. They are apparently an excellent starting approxi-
mation for precise modeling the empirical PDFs.

These distributions can be modified further to describe two other well established stylized facts which are

(iii) decaying long ranged volatility-volatility correlations (volatility clustering) and (iv) return-volatility correlations (leverage effect). 
The long ranged correlations occur, since the value $\eta^{2}$ in the representation

$$
\xi_{\mathbf{i}}=\omega_{\mathbf{i}} \psi_{\mathbf{i}}\left|\frac{\alpha}{\eta^{2}}\right|^{\frac{1}{2}}
$$

is common for all the increments $\xi_{i}$. Decay of the correlations $\Rightarrow$ take different $\eta$ 's for different groups of the $\psi_{i}$ 's.

\section{ANALOGY WITH ISING MODEL:}

The components $\psi_{i}$ with the same denominator $\eta^{2}$ can be treated as domains of spins aligned in the same direction. The usual probability:

$$
\mathbf{w}\left[\sigma_{1}, \ldots, \sigma_{\mathbf{n}} \mid \beta\right]=\mathbf{N} \exp \left(-\beta \sum_{\mathbf{i}=1}^{\mathbf{n}-1} \sigma_{\mathbf{i}} \sigma_{\mathbf{i}+\mathbf{1}}\right)
$$

where $\sigma_{i}= \pm 1$. 
The normalization constant:

$$
1 / \mathbf{N}=2(2 \cosh (\beta))^{\mathbf{n}-1}
$$

The correlation of $\left|\xi_{i}\right|$ and $\left|\xi_{k}\right|$ equals the old value provided that these increments belong to the same domain and zero otherwise.

The probability to get the $\xi_{i}$ and $\xi_{k}$ within the same domain:

$$
\mathbf{w}_{1}=\mathbf{e}^{-\gamma \mathbf{l}}
$$

where

$$
\mathbf{e}^{-\gamma}=\frac{\mathrm{e}^{-\beta}}{\mathbf{e}^{\beta}+\mathbf{e}^{-\beta}}<1
$$

and $l=i-k$. $\operatorname{Corr}\left[\left|\xi_{i}\right|,\left|\xi_{k}\right|\right]$ has the old form multiplied by $w_{l}$. Superposition with different $\gamma$ is possible. 
To incorporate the leverage effect $\Rightarrow \omega_{i}$ depending on the signs $\epsilon_{i-p}=\operatorname{sign}\left(\xi_{i-p}\right)$ of the lagged increments $(p=1,2, \ldots)$. The function $\omega_{i}=\chi\left(\epsilon_{i-1}, \epsilon_{i-2}, \ldots\right)>0$ is defined by

$$
\omega_{\mathbf{i}}=\mathbf{C}\left(1-\rho \frac{1-\nu}{\nu} \sum_{\mathbf{p}=1}^{\infty} \nu^{\mathbf{p}_{\epsilon_{i}-\mathbf{p}}}\right)^{2}
$$

We require $0<\nu<1$ to ensure the convergence of the series.

Negative recent returns $\epsilon_{i-p}=-1(p=1,2, \ldots)$ increase the volatility, so $\rho>0$.

The normalization condition: $\mathrm{E}\left[\omega_{i}\right]=1$, so $C=\left(1+\rho^{2} \frac{1-\nu}{1+\nu}\right)^{-2}$.

The value of $\rho$ gives the overall strength of the leverage effect, while $\log (1 / \nu)$ is the decay time. 
Taking the volatility clustering and the leverage effect into account, we obtain:

$$
\begin{aligned}
\operatorname{Corr}\left[\left|\xi_{i}\right|,\left|\xi_{k}\right|\right] & =\frac{2}{\pi} \frac{\left(1-\frac{2}{\pi}\right) g_{l} w_{l}+\frac{2}{\pi}\left(g_{l}-1\right)}{g_{0}-\frac{4}{\pi^{2}}} \\
\operatorname{Corr}\left[\left|\xi_{i}\right|, \xi_{k}\right] & =\frac{2}{\pi} \frac{\left(1-\frac{2}{\pi}\right) w_{l}+\frac{2}{\pi}}{\sqrt{\left(g_{0}-\frac{4}{\pi^{2}}\right) g_{0}}} h_{l}
\end{aligned}
$$

where $g_{l}=\mathrm{E}\left[\omega_{i} \omega_{k}\right], h_{l}=\mathrm{E}\left[\omega_{i} \epsilon_{k} \omega_{k}\right]$, and $l=i-k \geq 0$. We have

$$
\left(1+\rho^{2} \frac{1-\nu}{1+\nu}\right)^{2} g_{l}=\left(1+\rho^{2} \frac{1-\nu}{1+\nu}\right)^{2}+4 \rho^{2} \frac{1-\nu}{1+\nu} \nu^{l}+4 \rho^{4} \frac{\nu^{2}(1-\nu)^{3}}{(1+\nu)\left(1-\nu^{4}\right)} \nu^{2 l}
$$

$$
\left(1+\rho^{2} \frac{1-\nu}{1+\nu}\right) h_{l}=-2 \rho \frac{1-\nu}{\nu} \nu^{l}
$$




\section{Deviations from Gaussian random walk: Autocorrelations}

The empirical correlation functions for the daily quotes of the DJIA are fitted

$$
w_{l}=c_{1} e^{-\gamma_{1} l}+c_{2} e^{-\gamma_{2} l} \quad l>0
$$

with $c_{1}=0.18, c_{2}=0.08, \gamma_{1}=1 / 1200$, and $\gamma_{2}=1 / 233$ (else $c_{0}=0.74$ and $\gamma_{0}=+\infty$, so that $\sum_{m=0}^{2} c_{m}=1$ ), also $\rho=1$ and $\nu=\exp (-1 / 16)$.

Correlation functions ver-

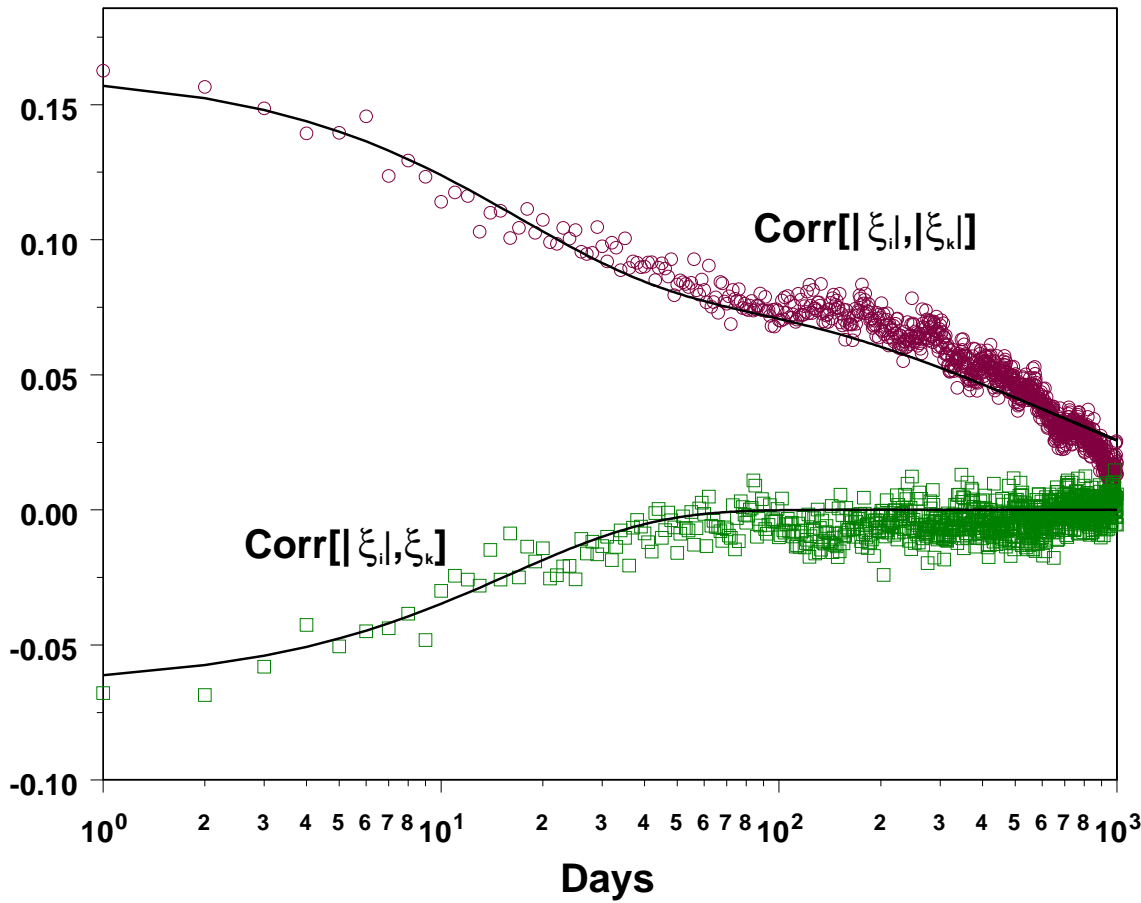
sus time lag $l=i-k$ between two increments $\xi_{i}$ and $\xi_{k}$. The empirical data are constructed using the daily quotes of the DOW Jones 30 Industrial Average. The volatility clustering (circles) and the leverage effect (boxes) are clearly seen. The data are compared to predictions of the Student multivariate PDF shown by the solid lines. 


\section{REALISTIC MULTIVARIATE STUDENT PDF}

$$
\begin{aligned}
\mathbf{S}_{\mathbf{n}}^{\alpha}\left(\xi_{\mathbf{n}}, \ldots, \xi_{1}\right)_{\mathbf{F}} & =\sum_{\mathbf{m}=\mathbf{0}}^{\mathbf{2}} \mathbf{c}_{\mathbf{m}} \sum_{\mathbf{s}=\mathbf{0}}^{\mathbf{n}-\mathbf{1}} \frac{\exp \left(-\beta_{\mathbf{m}}(\mathbf{n}-2 \mathrm{~s}-\mathbf{1})\right)}{\left(2 \cosh \left(\beta_{\mathbf{m}}\right)\right)^{\mathbf{n}-1}} \\
& \left.\times \sum_{\mathbf{n}_{\mathbf{s}}, \ldots, \mathbf{n}_{\mathbf{0}} \mathbf{f}=\mathbf{1}} \prod_{\mathbf{n}_{\mathbf{f}}-\mathbf{n}_{\mathbf{f}-\mathbf{1}}}^{\mathrm{s}+\mathbf{1}} \mathbf{S}_{\mathbf{n}_{\mathbf{f}}-\mathbf{1}}^{\alpha}, \ldots, \xi_{\mathbf{n}_{\mathbf{f}-1}}\right)
\end{aligned}
$$

where $n_{s+1}=n+1, n \geq n_{s}>\ldots>n_{1} \geq 2$, and $n_{0}=1$. The calculation time for computers increase exponentialy with $n$. The straightforward calculation of the $n=13$ PDF takes $\sim 1$ Min. The marginal probability of the PDF (9) is a PDF (9) again:

$$
\int \mathbf{S}_{\mathbf{n}}^{\alpha}\left(\xi_{\mathbf{n}}, \ldots, \xi_{\mathbf{1}}\right)_{\mathbf{F}} \prod_{\mathbf{i}=\mathbf{k}+\mathbf{1}}^{\mathbf{n}} \mathrm{d} \xi_{\mathbf{i}}=\mathbf{S}_{\mathbf{k}}^{\alpha}\left(\xi_{\mathbf{k}}, \ldots, \xi_{1}\right)_{\mathbf{F}}
$$


The value of $c_{0}=0.74$ turned out to be unexpectedly large. It indicates that

Three quarters of the empirical PDF consist of the product of the Student univariate PDFs.

The product does converge to the Gaussian PDF due to the central limit theorem. One should thus verify that the proposed PDF nevertheless supports the approximate scaling observed for financial time series.

A qualitative explanation: the Levy stable PDF fits well the central part of the empirical distributions and therefore of the Student PDF, providing an exceptionally slow convergence to the Gaussian PDF. 
The realistic PDF is constructed for the daily increments. There are no a priori reasons to believe that the daily scale is more appropriate than other time scales.

\section{The criterion for selection of the best time interval is the quality of the fit of the empirical univariate PDF.}

It is seen from Fig. 4 that e.g. $\Delta t=13$ days would be an inaccurate choice. At the same time, the excellent fit of the daily empirical PDF indicates that the assumption

$$
\Delta t=1 \text { day }
$$

on which the realistic PDF is based is quite reasonable. 


\section{Deviations from Gaussian random walk: Approximate scaling}

\section{Scaled univariate Student PDFs}

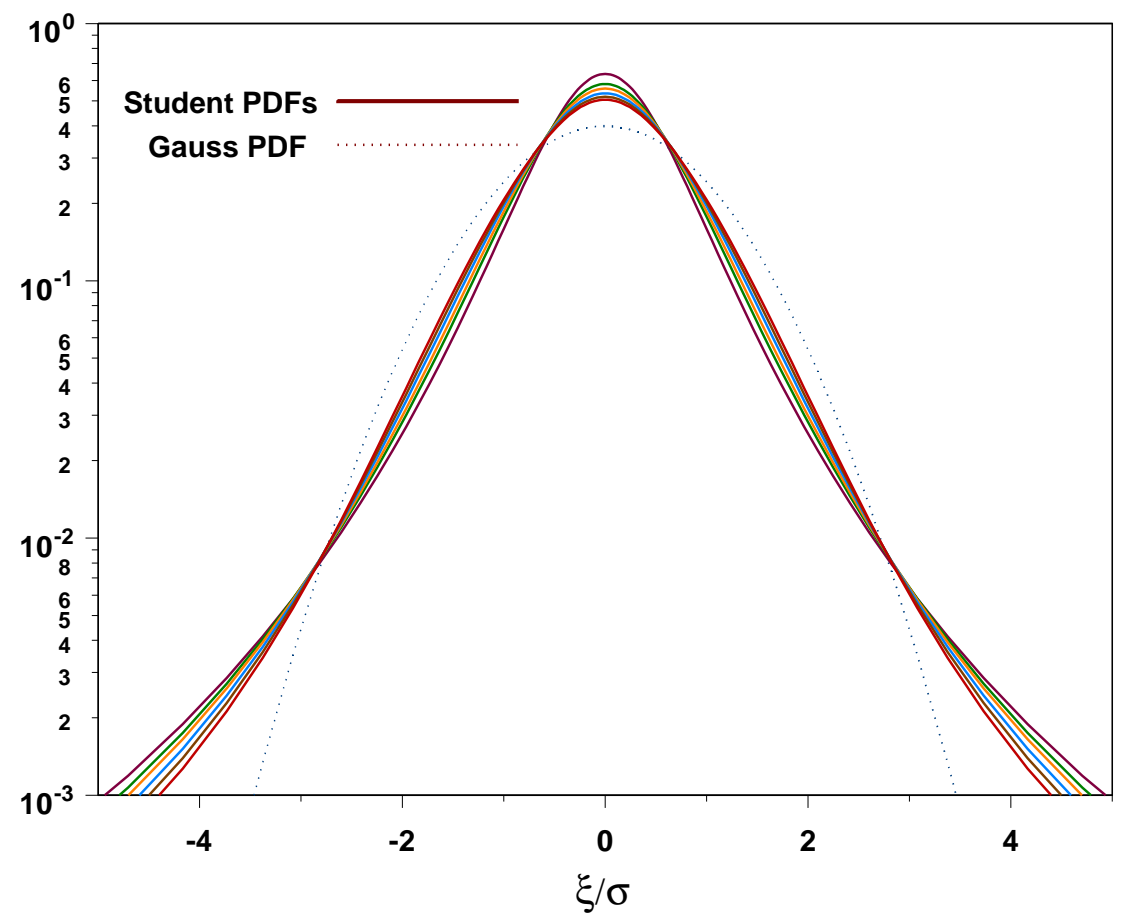

The $\alpha=3$ scaled Student PDF for $n=1,2,3,5,8$, and 13 (solid lines) as compared to the Gaussian PDF (short-dashed line). The lower vales of the $n$ correspond to the higher values of the PDF at the origin $\xi=0$ and to the lower values at the high absolute values of the $\xi$. The convergence to the Gaussian PDF with increasing the $n$ is slow. The value of $\xi$ is the noise added to the path followed by the index and $\sigma^{2}$ is variance of the $\xi$. 
Deviations from Gaussian random walk: Approximate scaling

Scaled $n=13$ distributions based on the realistic modified multivariate Student PDF as compared to the empirical PDF for $100+$ years of the DJIA daily quotes

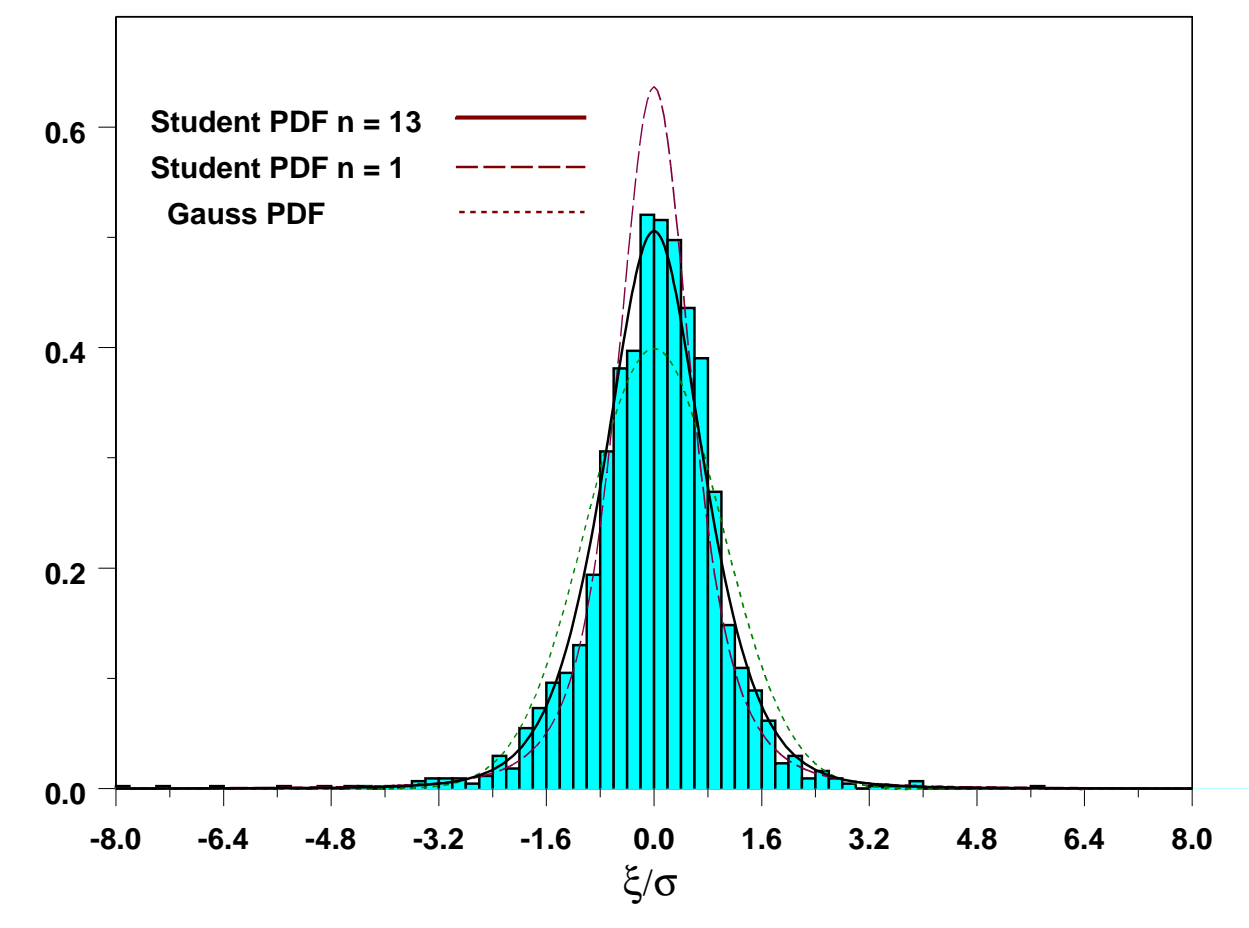

The $n=13$ and $n=1$ Student and Gaussian PDFs as compared to the hystogram for the $100+$ years of quotes of the DOW Jones 30 Industrial Average. The value of $\xi$ is the increment (noice) added to the path followed by the index value, $\sigma^{2}=\operatorname{Var}[\xi]$. The common scale of the distributions is fixed by fitting the variance of the $\xi$. The solid line stands for the $n=13$ modified multivariate Student PDF, the longdashed line stands for the $n=1$ Student PDF, and the shortdashed line denotes the Gaussian PDF. 
Ensemble of uncorrelated stocks $\Rightarrow$ marginal probabilities

$$
\text { Single index } \Rightarrow \text { time averages } \Rightarrow \text { conditional probabilities }
$$

The above discussion refers to the marginal probabilities. If no correlations exist, the marginal probabilities coincide with the conditional probabilities.

Ergodic hypothesis is valid, since all the val-
ues $\gamma_{k}$ and $\ln (1 / \nu)$ are distinct from zero, so
the correlation lengths are all finite.

It means that the time averages are equivalent to the ensemble averages, provided that the time scale used for the estimation is much longer than the largest correlation length $\left(1 / \gamma_{1} \sim 5\right.$ calendar years).

At smaller time scales, however, the distinction between these two types of the probabilities can be significant. 


\section{Conditional PDFs}

The conditional PDF of the increments $\xi_{n}, \ldots, \xi_{k+1} \mid \xi_{k}, \ldots, \xi_{1}$ equals

$$
\mathbf{S}_{\mathbf{n}}^{\alpha}\left(\xi_{\mathbf{n}}, \ldots, \xi_{\mathbf{k}+1} \mid \xi_{\mathbf{k}}, \ldots, \xi_{1}\right)_{\mathbf{F}}=\frac{\mathbf{S}_{\mathbf{n}}^{\alpha}\left(\xi_{\mathbf{n}}, \ldots, \xi_{1}\right)_{\mathbf{F}}}{\mathbf{S}_{\mathbf{k}}^{\alpha}\left(\xi_{\mathbf{k}}, \ldots, \xi_{1}\right)_{\mathbf{F}}}
$$

It is normalized to unity. For $i>k$

$$
\begin{aligned}
\mathrm{E}\left[\xi_{i} \mid \xi_{k}, \ldots, \xi_{1}\right]_{F}= & 0 \\
\operatorname{Var}\left[\xi_{i} \mid \xi_{k}, \ldots, \xi_{1}\right]_{F}= & 2^{-(i-k)} \sum_{\epsilon_{i}, \ldots, \epsilon_{k+1}} \sum_{m=0}^{2} c_{m} \sum_{v=1}^{i} \frac{\exp \left(-\beta_{m}(i-v-\kappa)\right)}{\left(2 \cosh \left(\beta_{m}\right)\right)^{i-v+\kappa}} \\
& \times \omega_{i}^{2} \frac{\alpha}{\alpha-2+\kappa_{1}}\left(1+\frac{1}{\alpha} \sum_{p=v}^{k} \omega_{p}^{-2} \xi_{p}^{2}\right)
\end{aligned}
$$

where $\kappa=\min (1, v-1)$, and $\kappa_{1}=\max (0, k+1-v)$. 


\section{Conditional PDFs}

The conditional volatility equals

$$
\sigma_{\mathbf{i}-\mathbf{1}}^{\mathbf{2}}=\operatorname{Var}\left[\xi_{\mathbf{i}} \mid \xi_{\mathbf{i}-1}, \ldots, \xi_{\mathbf{1}}\right]_{\mathbf{F}}
$$

while the old expression

$$
\sigma_{\mathbf{i}}^{2}=\omega_{\mathbf{i}}^{2} \frac{\alpha}{\alpha-2}
$$

can be referred to as the marginal (unconditional) volatility. The ensemble average of (12) gives (13).

It is hard to distinguish empirically between the increments $\xi_{i}$ and returns $\Delta_{i}$. The asymptotic $d W \sim d \xi / \xi^{4} \sim e^{-3 \Delta} d \Delta$ implies $\mathrm{E}\left[S_{n}\right]<\infty$, and $\operatorname{Var}\left[S_{n}\right]=\infty$, while $d W \sim d \Delta / \Delta^{4} \sim d \xi /\left(\xi \ln ^{4} \xi\right)$ would cause more severe theoretical problems: $\mathrm{E}\left[S_{n}\right]=\operatorname{Var}\left[S_{n}\right]=\infty$. The asymptotic behavior $\sim d \xi / \xi^{4}$ refers naturaly to the increments $\xi_{i}$. 
Return on an asset per time interval $\left[t_{i}, t_{i-1}\right]$ :

$$
\Delta_{\mathbf{i}} \equiv \ln \mathbf{S}_{\mathbf{i}} / \mathbf{S}_{\mathbf{i}-\mathbf{1}}
$$

The increments $\xi_{i} \sim 10^{-2}$ and the drift rate $\mu$ are small, and so $\Delta_{i} \simeq \xi_{i}$ to a first approximation.

The Ito's lemma can be applied to $\ln S(t)$ provided that $\mathrm{E}\left[(d \xi(t))^{n}\right]<\infty$ for all $n$. In our case, the asymptotic distributions of the increments are such that $\mathrm{E}\left[(d \xi(t))^{2 n}\right]=\infty$ for $n \geq 2$. Equation

$$
\boldsymbol{\Delta}_{\mathbf{i}}=\ln \left(\mathbf{1}+\mu+\xi_{\mathbf{i}}\right)
$$

cannot be simplified further by expanding the logarithm in power series of the $\xi_{i}$. 
The multivariate PDF of returns:

$$
\mathrm{d} \mathbf{W}\left[\Delta_{\mathbf{i}}\right]=\mathbf{S}_{\mathbf{n}}^{\alpha}\left(\xi_{\mathbf{i}}\right)_{\mathbf{F}} \prod_{\mathbf{i}=1}^{\mathbf{n}} \mathrm{d} \xi_{\mathbf{i}}
$$

where $\xi_{i}$.

The knowledge of the multivariate PDF provides, in principle, the most complete information on the future market behavior.

A simple check: Fair value of a market index at $t=t_{k}$

$$
\mathrm{E}\left[\mathbf{S}_{\mathbf{n}}\right]=\mathbf{S}_{\mathbf{k}} \mathrm{E}\left[\exp \left(\sum_{\mathbf{i}=\mathbf{k}+\mathbf{1}}^{\mathbf{n}} \boldsymbol{\Delta}_{\mathbf{i}}\right) \mid \xi_{\mathrm{k}}, \ldots, \xi_{1}\right]_{\mathbf{F}}=(\mathbf{1}+\mathbf{r})^{\mathbf{l}} \mathbf{S}_{\mathbf{k}}
$$

where $\mu=r$ is the risk-free discount rate corresponding to the expiration date $t_{n}$. The result has the conventional form. The dispersion of $S_{n}$, however, diverges: $\operatorname{Var}\left[S_{n} \mid \xi_{k}, \ldots, \xi_{1}\right]_{F}=\infty$. 
Pricing of the Log contracts: The fair value of a long position, $L=\ln S_{n} / S_{k}$, is defined by:

$$
\mathrm{E}[\mathrm{L}]=\mathrm{E}\left[\sum_{\mathrm{i}=\mathrm{k}+\mathbf{1}}^{\mathrm{n}} \Delta_{\mathrm{i}} \mid \xi_{\mathrm{k}}, \ldots, \xi_{1}\right]_{\mathrm{F}}
$$

Using the conditional PDF, we obtain:

$$
\mathrm{E}\left[\sum_{\mathrm{i}=\mathrm{k}+1}^{\mathrm{n}} \boldsymbol{\Delta}_{\mathrm{i}} \mid \xi_{\mathrm{k}}, \ldots, \xi_{1}\right]_{\mathrm{F}} \simeq 2^{-1} \sum_{\epsilon_{\mathrm{n}}, \ldots, \epsilon_{\mathrm{k}+1}} \sum_{\mathrm{i}=\mathrm{k}+1}^{\mathrm{n}}\left(\mathrm{r}-\frac{1}{2} \sigma_{\mathrm{ik}}^{2}\right) .
$$

The result depends on the lagged increments through

$$
\sigma_{\mathrm{ik}}^{2}=\operatorname{Var}\left[\xi_{\mathrm{i}} \mid \xi_{\mathrm{k}}, \ldots, \xi_{1}\right]_{\mathrm{F}} .
$$

The pricing of the Log contracts is well defined both with respect to the fair value and its dispersion. 


\section{CONCLUSION:}

- The asymptotic behavior is important for the correct estimates of financial risks. The use of the multivariate Student PDF gives more precise idea on the involved risks and, in particular, on those ones connected to large market fluctuations.

$\checkmark$ The following four stylized facts

(i) heavy tails,

(ii) approximate scaling of the returns,

(iii) volatility clustering,

(iv) leverage effect

are well established empirically, but missing in the Gaussian random walk model. We constructed the multivariate PDFs of incremenets and returns for financial markets, that take approximately those four effects into account.

- The model can be useful for more accurate pricing derivative securities and risk management. 